\title{
Sooting tendencies of diesel fuel component mixtures follow a linear mixing rule
}

\author{
Zhanhong Xiang ${ }^{1}$, Karnsiree Chen ${ }^{1}$, Charles S. McEnally ${ }^{1, *}$, Lisa D. Pfefferle ${ }^{1}$ \\ ${ }^{1}$ Department of Chemical and Environmental Engineering, Yale University, New Haven CT 06520 USA
}

\begin{abstract}
With the growing importance of climate change, soot emissions from engines have been receiving increasing attention since black carbon is the second largest source of global warming. A sooting tendency can be used to quantify the extent of soot formation in a combustion device for a given fuel molecule, and therefore to quantify the soot reduction benefits of alternative fuels. However real fuels are complex mixtures of multiple components. In this work, we have used experimental methods to investigate how the sooting tendency of a blended fuel mixture is related to the sooting tendencies of the individual components. A test matrix was formulated that includes sixteen mixtures of six components that are representative of the main categories of hydrocarbons in diesel (eicosane (ECO) for alkanes, isocetane (ICE) for isoalkanes, butylcyclohexane $(\mathrm{BCH})$ for cycloalkanes, 1-methylnaphthalene (1MN) for aromatics, tetralin for naphthoaromatics, and methyl-decanoate (MDC) for oxygenates). Most of the mixtures contain three to five components. The sooting tendency of each mixture was characterized by yield sooting index (YSI), which is based on the soot yield when a methane/air nonpremixed flame is doped with $1000 \mathrm{ppm}$ of the test fuel. The YSIs were measured experimentally. The results show that the blending behavior is linear, i.e., the YSI of the mixtures is the mole-fraction-weighted average of the component YSIs. Experimental results have shown that the sooting tendency of a fuel mixture can be accurately estimated as the linear combination of the individual components. In addition, mass density of the mixtures is also measured, and a linear blending rule is applied to test whether mixing rules exist for mass density of diesel mixtures in this study. Results also have shown that the mixing rule tested in this study is valid and mass density of a mixture can be accurately estimated from the linear combination of the individual components.
\end{abstract}

Keywords: diesel mixtures; soot emissions; yield sooting index (YSI); mass density; blending rules.

Preprint submitted to Chemxriv

January 82022

*Corresponding author. Charles S. McEnally

(charles.mcenally@yale.edu) 


\section{Introduction}

The ongoing transition from fossil fuels to biofuels and e-fuels presents a unique opportunity to improve performance and reduce emissions [1, 2]. However, the chemical space of potential fuels is enormous, so achieving this goal requires procedures that can accurately predict the properties of fuels before they are synthesized. Since real fuels are typically mixtures, mixing rules are necessary that can estimate the properties of mixtures from the properties of their individual components. This paper tests some simple mixing rules for mass density and sooting tendency of diesel fuel mixtures.

Mass density $(\rho)$ is a fundamental physical property of a fuel that determines its energy content. It is frequently used as a target parameter when formulating fuel surrogates $[3,4]$. It may also impact soot formation in advanced engine concepts [5]. Mixing rules are more easily formulated for specific volume $(v)$, which is the inverse of $\rho$, since $v$ is a property that increases with more substance. For an ideal solution, the specific volume of the mixture $v_{\text {mix }}$ is given by

$$
v_{\text {mix }}=\sum_{j} w_{j} \times v_{j}
$$

where $w_{j}$ and $v_{j}$ are the mass fraction and the specific volume of component $j$, and the sum is over all the components in the solution [6]. Equation 1 is readily derived from the assumption that the total volume of the solution is the sum of the volumes of the individual molecules; see Supplemental Information (SI) A. The weighting factor is $w$ since specific volume is a massic quantity (volume/mass); it would be mole fraction $x$ for the corresponding molar quantity molar volume (volume/mole). Equation 1 can be re-written for the mass density of the mixture $\rho_{\text {mix }}$ as

$$
\frac{1}{\rho_{\mathrm{mix}}}=\sum_{j} w_{j} \times \frac{1}{\rho_{j}}
$$

where $\rho_{j}$ is the mass density of component $j$.

Several alternative approaches can be used to estimate $\rho$ for mixtures. A simple engineering mixing rule is

$$
\rho_{\text {mix }}=\sum_{j} \varphi_{j} \times \rho_{j}
$$

where $\varphi_{j}$ is the volume fraction of component $j$. This rule has been used in several surrogate studies $[7,8]$. Since $\rho$ is a thermodynamic property, it can also be calculated with equations of state (EoSs) [9]. EoS calculations are more precise and can estimate $\rho$ at variable temperature and pressure, but they also require extensive parameters for each species, which limits the range of molecules that can be considered. For example, the National Institute of Standards and Technology (NIST) REFPROP software supports less than 130 hydrocarbons and is limited to mixtures with 20 or fewer components [10].

Sooting tendency is a laboratory-scale property that indicates how readily a fuel produces soot particles due to its chemical composition [11]. Soot emissions are the second largest source of global warming [12], and they contribute to ambient fine particulates that cause millions of deaths each year [13]. Alternative fuels can greatly ameliorate these problems given that soot formation depends strongly on fuel composition [14]. Sooting tendency is traditionally measured with the ASTM D1322 smoke point test [15], which measures the height of a wickburner flame at the point when soot first breaks through the tip. More recently, we have developed an alternative definition based on the soot yield in fueldoped flames, which we call yield sooting index (YSI) [16]. YSI requires orders of magnitude less sample volume than smoke point $(100 \mu \mathrm{L}$ versus $10 \mathrm{~mL})$, which makes it useful for developing new fuel molecules. We have applied it to custom-synthesized alternative fuels such as dioxolanes and polyoxymethylene ethers where the available sample volumes were insufficient to measure smoke point $[17,18]$

Sooting tendency is a derived combustion property, not a fundamental physical property like $\rho$, so mixing rules for it are necessarily empirical. Several studies have found good agreement between linear mixing rules and measured smoke points [e.g., 19, 20]. Therefore, we propose that the yield-based sooting tendency of a mixture $\mathrm{YSI}_{\text {mix }}$ is given by

$$
\mathrm{YSI}_{\mathrm{mix}}=\sum_{j} x_{j} \times \mathrm{YSI}_{j}
$$

where $x_{j}$ and $\mathrm{YSI}_{j}$ are the mole fraction and the sooting tendency of component $j$. Mole fraction is used as the weighting factor since the YSI protocol adds a fixed mole fraction to the base flame. We have used Eq. (4) previously [21], but this is the first study to systematically evaluate it.

In this study the linear mixing rules were tested for mixtures representative of diesel fuels. First, we defined a set of mixtures containing various proportions of six components that represent the main chemical families in diesel fuel. The mixture matrix followed a formal experimental design to ensure that it sampled the full chemical space possible with six components. The $\rho$ of these mixtures were measured, then the results were fit to Eq. (2). Similarly, the YSIs of the mixtures were measured, then fit to Eq. (4). 


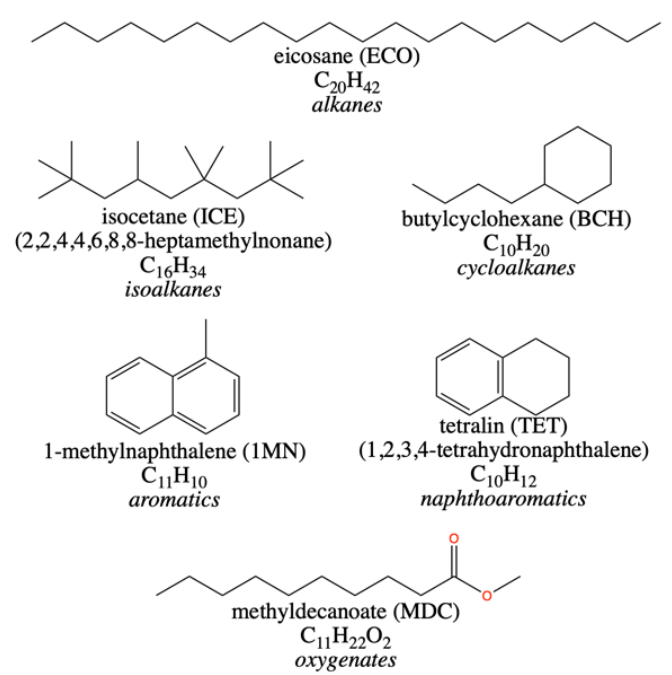

Fig. 1. The structures, molecular formulas, and chemical families of the mixture components.

\begin{tabular}{|c|c|c|c|c|c|c|}
\hline Mixture \# & $\boldsymbol{X}_{\mathrm{ECO}}$ & $\boldsymbol{X}_{\mathrm{BCH}}$ & $\boldsymbol{X}_{\mathrm{TET}}$ & $\boldsymbol{X}_{\mathrm{MDC}}$ & $\boldsymbol{X}_{\text {ICE }}$ & $\boldsymbol{X}_{1 \mathrm{MN}}$ \\
\hline 1 & 0 & 0 & 0 & 0 & 0 & 1.0000 \\
\hline 2 & 0.3238 & 0 & 0 & 0 & 0.3415 & 0.3347 \\
\hline 3 & & 0.4931 & 0 & 0 & 0.5069 & 0 \\
\hline 4 & 0.3265 & 0.6735 & 0 & 0 & 0 & 0 \\
\hline 5 & 0 & 0 & 0.4923 & 0 & 0.5077 & 0 \\
\hline 6 & 0.2456 & 0 & 0.7544 & 0 & 0 & 0 \\
\hline 7 & 0 & 0.3308 & 0.3316 & 0 & 0 & 0.3376 \\
\hline 8 & 0.1925 & 0.1987 & 0.1991 & 0 & 0.2057 & 0.2040 \\
\hline 9 & 0 & 0 & 0 & 1.0000 & 0 & 0 \\
\hline 10 & 0.2462 & 0 & 0 & 0.3699 & 0.3838 & 0 \\
\hline 11 & 0 & 0.2480 & 0 & 0.2432 & 0.2577 & 0.2512 \\
\hline 12 & 0.2461 & 0.2510 & 0 & 0.2482 & 0 & 0.2546 \\
\hline 13 & 0 & 0 & 0.2447 & 0.2481 & 0.2552 & 0.2519 \\
\hline 14 & 0.2488 & 0 & 0.2482 & 0.2499 & 0 & 0.2532 \\
\hline 15 & 0 & 0.3324 & 0.3335 & 0.3341 & 0 & 0 \\
\hline 16 & 0.1968 & 0.1992 & 0.1986 & 0.2001 & 0.2054 & 0 \\
\hline
\end{tabular}

Fig. 2. The mole fractions of each component in each mixture.

\section{Procedures}

\subsection{Mixture Design}

In this study, we prepared sixteen mixtures of six components that are representative of the main categories of hydrocarbons in diesel fuels: eicosane (ECO) for alkanes, isocetane (ICE) for isoalkanes, butylcyclohexane $(\mathrm{BCH})$ for cycloalkanes, 1methylnaphthalene $(1 \mathrm{MN})$ for aromatics, tetralin (TET) for naphthoaromatics, and methyldecanoate (MDC) for oxygenates. Fig. 1 shows the chemical structures of these components. The first five components are pure hydrocarbons that were selected from the surrogate fuel palette defined in Ref. [3]. The last component (MDC) is an oxygenate that represents the methyl esters found in biodiesel fuels [31]. MDC was selected because it has a diesel-like carbon number $(\mathrm{C} 11)$, and detailed chemical kinetic mechanisms are available for it [32].
The compositions of the mixtures followed a modified $2 \mathrm{IV}^{6-2}$ fractional factorial experimental design [29] to ensure that the experiments sampled the full chemical space possible with six components, and that they systematically tested all possible non-linear interactions between the components. To illustrate how this works, Fig. 2 shows the mole fractions $(x)$ of each component in each mixture. (This figure is intended to illustrate the structure of the mixture matrix; SI B provides more legible tables of the mixture composition.) Cells where $x \neq 0$ are highlighted in blue. If we consider the columns for TET and MDC, the first tetrad of mixtures (numbers 1 to 4) contains neither of these components, the second tetrad (5 to 8) contains TET but not MDC, the third tetrad (9 to 12) contains MDC but not TET, and the fourth tetrad (13 to 16) contains both components. Thus, the difference between the second and first tetrads indicates the effect of TET in the absence of MDC, the difference between the third and first tetrads indicates the effect of MDC in the absence of TET, and the difference between the fourth and first tetrads indicates the effect of both components together; if this latter effect equals the sum of the first two, then there is no nonlinear interaction between TET and MDC. The experimental design guarantees that this same structure of tetrads applies to all other pairs of components.

\subsection{Mixture Preparation}

SI C lists the sources and purities of the mixture components. The mixtures were prepared gravimetrically by adding each component to $2 \mathrm{~mL}$ vials with septa in the caps. ECO is a solid at room temperature, but it dissolved readily in the other components. The mass of each component was measured with a Mettler Toledo NewClassic MF analytical balance $(0.1 \mathrm{mg}$ precision), mass fractions were calculated from these masses, and then mole fractions were calculated from the known molecular mass of each component. SI B lists the final mass fractions and mole fractions. The uncertainties in these values are estimated to be \pm 0.001 .

\subsection{Density Measurements}

Mass density was measured by dispensing $50 \mu \mathrm{L}$ of each mixture into an empty vial with a syringe (Hamilton Gastight 1705), then determining the mass of the dispensed liquid with the same balance used for mixture preparation. Each density was measured six times and then averaged. The systematic uncertainties are estimated to be $0.005 \mathrm{~g} / \mathrm{mL}$ based on densities of pure compounds compared to literature values, and the random uncertainties are estimated to be 0.009 $\mathrm{g} / \mathrm{mL}$ based on the standard deviation of measuring densities six times. Overall, we estimate that the uncertainty in the measured mixture density is $\pm 1 \%$. 


\subsection{YSI Measurements}

Sooting tendencies were measured using a yieldbased approach we developed previously [16]. The procedure used in this study is described in [21]. It consisted of three steps: (1) $1000 \mathrm{ppm}$ of MDC, $1 \mathrm{MN}$, and each mixture (mix) were separately doped into the fuel of a methane/air flame; (2) the maximum soot concentration was measured in each flame with lineof-sight spectral radiance (LSSR); and (3) these concentrations were rescaled into a yield sooting index (YSI) defined by:

$$
\begin{aligned}
\mathrm{YSI}_{\text {mix }}= & \left(\mathrm{YSI}_{1 M N}-\mathrm{YSI}_{\mathrm{MDC}}\right) \times \frac{\mathrm{LSSR}_{\mathrm{mix}}-\mathrm{LSSR}_{\mathrm{MDC}}}{\mathrm{LSSR}_{1 \mathrm{MN}}-\mathrm{LSSR}_{\mathrm{MDC}}}+ \\
\mathrm{YSI}_{\mathrm{MDC}} & \text { (5) }
\end{aligned}
$$

This rescaling removes sources of systematic uncertainty such as errors in the methane and air flowrates. Furthermore, it allows the new results to be quantitatively compared with a database that contains measured YSIs for hundreds of organic compounds [22].

We typically use n-heptane and toluene as the endpoint species (e.g., [21]), but in this case that would have required a large extrapolation since the YSIs of TET and especially $1 \mathrm{MN}$ are much larger than toluene (TET $\approx 400$ and $1 \mathrm{MN} \approx 650 \mathrm{vs}$. toluene $\approx 170$ ). Therefore, $1 \mathrm{MN}$ and MDC were chosen as upper and lower endpoints specific to this study that were guaranteed to bracket the diesel mixtures. The endpoint values $\left(\mathrm{YSI}_{1 \mathrm{MN}}\right.$ and $\mathrm{YSI}_{\mathrm{MDC}}$ ) were set to 620.1 and 60.1. These values were calculated with a group contribution model (GCM) [30] (see SI D). Predicted values were used instead of measured values because (1) the predicted values are close to the measured values (649.1 and 50.4), and (2) the GCM combines the results for hundreds of measurements and therefore provides better consistency with other species.

SI E shows a schematic diagram of the LSSR apparatus and SI F gives details of the specific burner $[23,24]$. The liquid diesel mixtures were injected into the gas-phase $\mathrm{CH}_{4} / \mathrm{N}_{2}$ fuel mixture with a syringe pump. SI G lists the liquid-phase flowrates corresponding to $1000 \mathrm{ppm}$ in the gas-phase for each mixture, and the property values [25] that were used to calculate them. Each mixture was injected for $600 \mathrm{~s}$ and the LSSR signal was averaged from 300 to $600 \mathrm{~s}$; SI H shows that the initial $300 \mathrm{~s}$ is adequate for the mixture to equilibrate with the walls of the fuel line and burner. The fuel lines were heated to $125^{\circ} \mathrm{C}$ and above, and the burner was heated to $170{ }^{\circ} \mathrm{C}$. SI I shows that at these temperatures the vapor pressures [28] of all the components were higher than their partial pressures. SI J shows that the LSSR signals of ECO, the least volatile component, increased linearly with the dopant mole fraction, which experimentally confirms that the components were not condensing in the fuel delivery system. Isooctane was included in each measurement set as an internal standard; SI K shows that the values were consistent over time and agreed with previous measurements [22]. SI L shows that the syringe pump stepper motor performed accurately.

Each YSI was measured three times and then averaged. The systematic uncertainty in YSI is estimated to be $\pm 1 \%$, mainly due to uncertainties in $\rho$ (which is needed to calculate the dopant flowrate). The random uncertainties were estimated to be $\pm 3 \%$ based on the standard deviation of the individual measurements. Overall, we estimate that the uncertainty in the measured mixture YSI is $\pm 4 \%$.

\section{Results}

\subsection{Mass Density}

SI $M$ lists the mass densities $\rho$ measured for each of the 16 diesel-like mixtures, and the specific volumes $v(=1 / \rho)$ calculated from them. The first objective of this study was to determine whether the linear mixing rules, Eqs. (1) and (2), can fit this data.

Eq (1) can be written in matrix form as

$$
v_{\text {mix }}=w v_{\text {comp }}
$$

where $v_{\text {mix }}$ is a $16 \times 1$ column vector that contains the measured specific volumes for each mixture, $\boldsymbol{w}$ is a 16 $\times 6$ matrix that contains the mass fraction of each component in each mixture, and $\boldsymbol{v}_{\text {comp }}$ is a $6 \times 1$ column vector that contains the specific volume of each component. We can regard $\boldsymbol{v}_{\text {mix }}$ as a known from the measurements (see SI M), $\boldsymbol{w}$ as a known from the mixture preparation (see SI B), and $\boldsymbol{v}_{\text {comp }}$ as an unknown. Eq. (6) does not have an exact solution since there are more mixtures than components and the $v_{\text {mix }}$ contain random measurement errors, but multiple linear least-squares regression can be used to find the $\boldsymbol{v}_{\text {comp }}$ that most closely solves it. We refer to the optimized component values in this solution as $v_{\text {opt }}$. This analysis was performed, and the results are listed in Table 1 (as densities). 
Table 1. Mass Densities of the Mixture Components. $\rho_{\text {lit }}$ are values obtained from the literature (averages of values from the vendor and [26] $\left(25^{\circ} \mathrm{C}\right)$, [27] $\left(20^{\circ} \mathrm{C}\right)$, and $\left.[28]\left(25^{\circ} \mathrm{C}\right)\right) . \rho_{\text {opt }}$ are values determined from the mixture measurements in this study.

\begin{tabular}{|c|r|r|r|}
\hline component & $\begin{array}{c}\rho_{\text {lit }} \\
(\mathrm{g} / \mathrm{mL})\end{array}$ & \multicolumn{1}{c|}{$\begin{array}{c}\rho_{\text {opt }} \\
(\mathrm{g} / \mathrm{mL})\end{array}$} & $\begin{array}{c}\rho_{\text {lit }}-\rho_{\text {opt }} \\
(\mathrm{g} / \mathrm{mL})\end{array}$ \\
\hline ECO & 0.8296 & 0.7821 & 0.0474 \\
\hline $\mathrm{ICE}$ & 0.7817 & 0.7855 & 0.0038 \\
\hline $\mathrm{BCH}$ & 0.7939 & 0.8023 & 0.0083 \\
\hline $\mathrm{TET}$ & 1.0381 & 1.0273 & 0.0108 \\
\hline $\mathrm{MDC}$ & 0.9679 & 0.9714 & 0.0035 \\
\hline
\end{tabular}

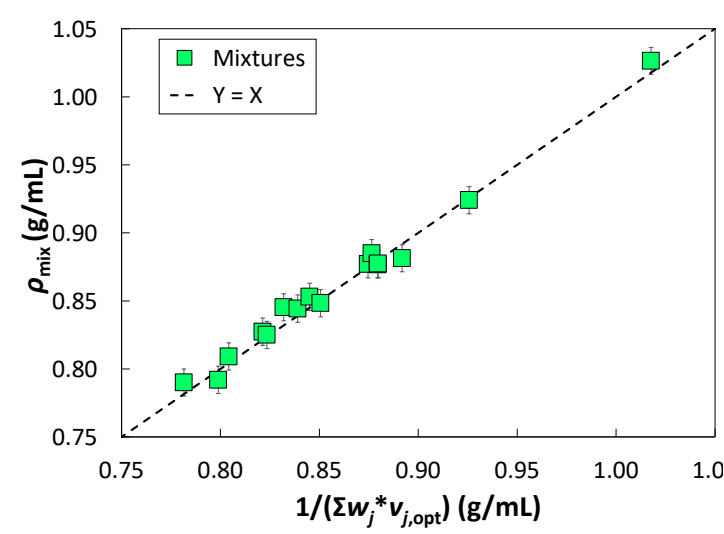

Fig. 3. Comparison of measured mass densities (vertical axis) to mass densities predicted with Eq (1) and the $\rho_{\text {opt }}$ in Table 1 (horizontal axis).

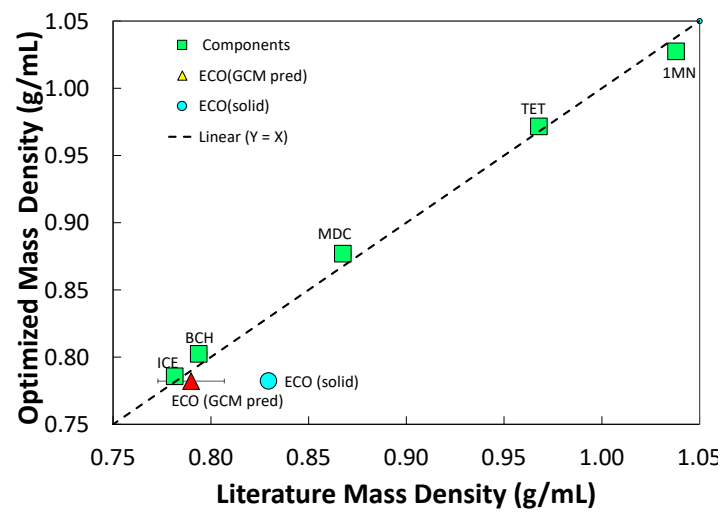

Fig. 4. Comparison of the component mass densities determined in this study (vertical axis) to mass densities from the literature (horizontal axis).
Fig. 3 compares the measured $\rho_{\text {mix }}$ (vertical axis) to the predicted $\rho_{\text {mix }}$ using the linear blending rule, Eq. (1), and the $\rho_{\text {opt }}$ (horizontal axis). The agreement is very good, which shows that the functional form of the linear blending rule can fit the measured data.

Table 1 also lists mass densities $\rho_{\text {lit }}$ for the components from the literature ([26] - [28]). Fig. 4 plots the $\rho_{\text {opt }}$ versus the $\rho_{\text {lit. }}$. For the components that are liquids at room temperature (ICE, $\mathrm{BCH}, 1 \mathrm{MN}$, TET, and MDC) the agreement is very good, which further validates the linear blending rule. On the other hand, the agreement is poor for ECO, which is a solid at room temperature. This shows that the solid phase $\rho$ is not an appropriate value for predicting the $\rho$ for a solid dissolved in a liquid. However, Fig 4 also shows a value of $\rho_{\text {lit }}$ calculated for ECO using the group contribution method (GCM) of Mathieu and Bouteloup [25], and in this case the agreement with the $\rho_{\text {opt }}$ is good. The GCM does not explicitly apply to a specific phase, but since it is extrapolating data measured for liquids to ECO, it generates predictions that appear to be appropriate for solids dissolved into liquids.

\subsection{Sooting Tendency (YSI)}

SI $\mathrm{N}$ lists the YSIs measured for each of the 16 diesel-like mixtures. The second objective of this study was to determine whether the linear mixing rule, Eq. (4), can fit this data.

Eq. (4) can be written in matrix form as

$$
\text { YSI }_{\text {mix }}=\mathbf{x} \text { YSI }_{\text {comp }}
$$

where $\mathbf{Y S I}_{\text {mix }}$ is a $16 \times 1$ column vector that contains the measured YSI for each mixture, $\mathbf{x}$ is a $16 \times 6$ matrix that contains the actual mole fraction of each component in each mixture, and YSI $_{\text {comp }}$ is a $6 \times 1$ column vector that contains the YSI of each component. Again, we regard YSI $_{\text {mix }}$ as a known from the measurements (see SI N), $\mathbf{x}$ as a known from the mixture preparation (see SI B), and $\mathbf{Y S I}_{\text {comp }}$ as an

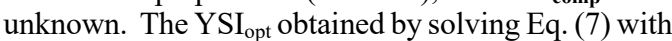
linear regression are listed in Table 2. 
Table 2. Sooting Tendencies of the Mixture Components. YSI $_{\mathrm{GCM}}$ are values calculated with the GCM [30] (see SI D). YSI meas $_{\text {are values measured }}$ for the components are pure compounds in earlier studies [30]. YSI $_{\text {opt }}$ are values determined from the mixture measurements in this study.

\begin{tabular}{|c|r|r|r|}
\hline component & \multicolumn{1}{|c|}{$\mathrm{YSI}_{\mathrm{GCM}}$} & \multicolumn{1}{c|}{$\mathrm{YSI}_{\text {meas }}$} & \multicolumn{1}{c|}{$\mathrm{YSI}_{\text {opt }}$} \\
\hline $\mathrm{ECO}$ & 122.2 & $\mathrm{~N} / \mathrm{A}$ & 137.34 \\
\hline $\mathrm{ICE}$ & 135.5 & 128.0 & 197.8 \\
\hline $\mathrm{BCH}$ & 77.0 & 76.8 & 88.41 \\
\hline $1 \mathrm{MN}$ & 620.1 & 649.1 & 645.07 \\
\hline $\mathrm{TET}$ & 396.3 & 336.0 & 397.78 \\
\hline $\mathrm{MDC}$ & 60.1 & 50.4 & 64.99 \\
\hline
\end{tabular}

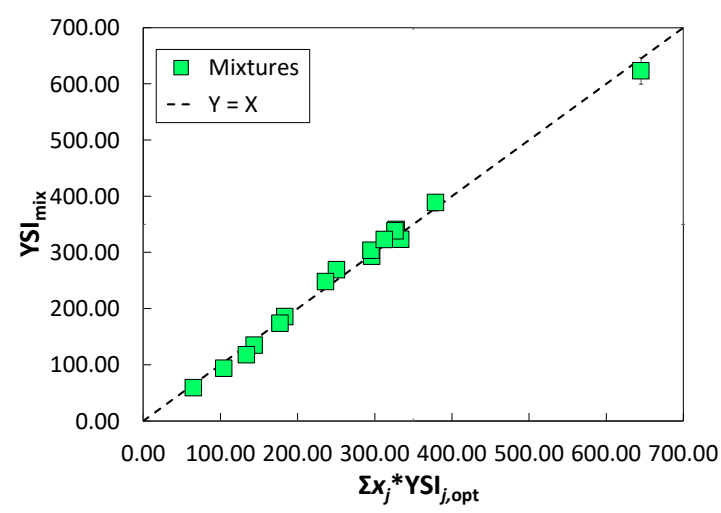

Fig. 5. Comparison of measured YSIs (vertical axis) to YSIs predicted Eq. (4) and the YSI $_{\text {opt }}$ in Table 2 (horizontal axis).

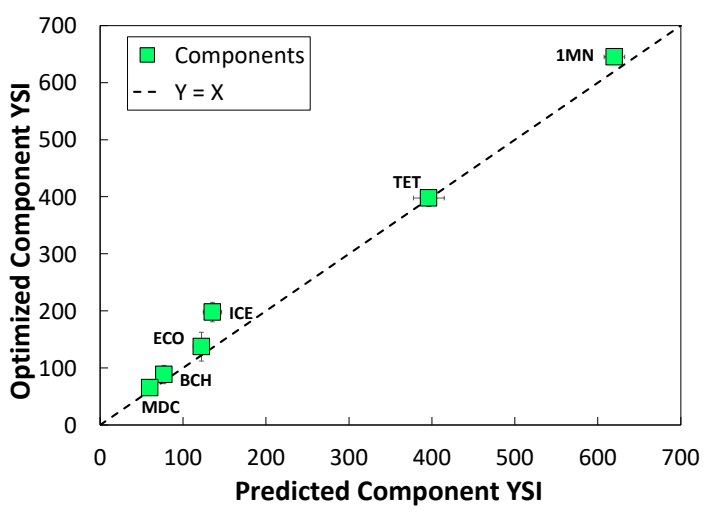

Fig. 6. Comparison of the component YSIs determined in this study (vertical axis) to YSIs predicted with the GCM (see SI D) (horizontal axis).
Fig. 5 compares the measured YSI $_{\text {mix }}$ (vertical axis) to the predicted $\mathrm{YSI}_{\text {mix }}$ using the linear blending rule, Eq. (4), and the YSI ${ }_{\text {opt }}$ (horizontal axis). The agreement is very good, which shows that the functional form of the linear blending rule can fit the measured data.

Table 2 also lists the sooting tendencies $\mathrm{YSI}_{\mathrm{GCM}}$ predicted for the components with the GCM from [30] (see SI D). Fig. 6 plots the $\mathrm{YSI}_{\mathrm{opt}}$ versus the $\mathrm{YSI}_{\mathrm{GCM}}$. The agreement is reasonable for all components, which further validates the linear blending rule.

\section{Conclusions}

In this study we prepared sixteen mixtures that contain six components representative of diesel fuels. The mixture compositions followed a formal experimental design to ensure they sampled the full chemical space possible with six components. Mass densities and sooting tendencies were measured for each mixture. Analysis of the results showed that the mass densities of diesel-like mixtures can be accurately estimated with a linear mixing rule for specific volume (the inverse of mass density) weighted by mass fraction. The group contribution method from [25] can be used to estimate appropriate component values for compounds that are solids at room temperature. Similarly, the sooting tendencies of diesel-like mixtures can be accurately estimated with a linear mixing rule weighted by mole fraction.

\section{Acknowledgements}

This work was based upon subcontract DE-A3608GO28308 from the Alliance for Sustainable Energy, LLC, Managing and Operating Contractor for the National Renewable Energy Laboratory for DOE.

\section{Supplementary material}

Supplemental material is available. 


\section{References}

[1] W. Leitner, J. Klankermayer, J, S. Pischinger, H. Pitsch, K. Kohse-Höinghaus, Advanced biofuels and beyond: chemistry solutions for propulsion and production, Angew. Chem. Int. Ed. 56 (2017) 54125452.

[2] J.P. Szybist, S. Busch, R.L. McCormick, J.A. Pihl, D.A. Splitter, M.A. Ratcliff, et al., What fuel properties enable higher thermal efficiency in sparkignited engines, Prog. Energy Combust. Sci. 82 (2021) 100876.

[3] C.J. Mueller, W.J. Cannella, T.J. Bruno, B. Bunting, H.D. Dettman, J.A. Franz, et al., Methodology for formulating diesel surrogate fuels with accurate compositional, ignition-quality, and volatility characteristics, Energy. Fuels 26 (2012) 3284-3303.

[4] S.M. Sarathy, A. Farooq, G.T. Kalghatgi, Recent progress in gasoline surrogate fuels, Prog. Energy Combust. Sci. 65 (2018) 67-108.

[5] Y. Zhang, A. Voice, Y. Pei, M. Traver, D. Cleary, A computational investigation of fuel chemical and physical properties effects on gasoline compression ignition in a heavy-duty diesel engine, J. Energy. Resources. Technol. 140 (2018) 102202. [6] F. Saryazdi, H. Motahhari, F.F. Schoeggl, S.D.Taylor, H.W. Yarranton, Density of hydrocarbon mixtures and bitumen diluted with solvents and dissolved gases, Energy Fuels 27 (2013) 3666-3678.

[7] A. Ahmed, G. Goteng, V.S.B. Shankar, K. AlQurashi, W.L. Roberts, S.M. Sarathy, A computational methodology for formulating gasoline surrogate fuels with accurate physical and chemical kinetic properties, Fuel 143 (2015) 290-300. [8] W. Yu, W. Yang, K. Tay, F. Zhao, An optimization method for formulating model-based jet fuel surrogate by emulating physical, gas phase chemical properties and threshold sooting index (TSI) of real jet fuel under engine relevant conditions, Combust. Flame 193 (2018) 192-217. [9] C. Nieto-Draghi, G. Fayet, B. Creton, X. Rozanska, P. Rotureau, J.-C. de Hemptinne, et al., A general guidebook for the theoretical prediction of physicochemical properties of chemicals for regulatory purposes, Chem. Rev. 115 (2015) 13093 13164.

[10] E.W. Lemmon, I.H. Bell, M.L. Huber, M.O. McLinden, NIST standard reference database 23: Reference fluid thermodynamic and transport properties-REFPROP, (2018),

https://doi.org/10.18434/T4/1502528.

[11] A. Gomez, G. Sidebotham, I. Glassman, Sooting behavior in temperature-controlled laminar diffusion flames, Combust. Flame 58 (1984) 45-57.

[12] T.C. Bond, S.J. Doherty, D.W. Fahey, P.M. Forster, T. Berntsen, B.J. DeAngelo, et al., Bounding the role of black carbon in the climate system: a scientific assessment, J. Geophys. Res. Atm. 118 (2013) 5380-5552.
[13] C.J.L. Murray, A.Y. Aravkin, P. Zheng, C. Abbafati, K.M. Abbas, M. Abbasi-Kangevari, et al, Global burden of 87 risk factors in 204 countries and territories, 1990-2019: a systematic analysis for the global burden of disease study 2019, Lancet 396 (2020) 1223-1249.

[14] P. Pepiot-Desjardins, H. Pitsch, R. Malhotra, S.R. Kirby, A.L. Boehman, Structural group analysis for soot reduction tendency of oxygenated fuels, Combust. Flame 154 (2008) 191-205.

[15] ASTM D1322-19 - Standard Test Method for Smoke Point of Kerosene and Aviation Turbine Fuel, ASTM International, (2019).

[16] C.S. McEnally, L.D. Pfefferle, Improved sooting tendency measurements for aromatic hydrocarbons and their implications for naphthalene formation pathways, Combust. Flame 148 (2007) 210-222.

[17] O. Staples, C.M. Moore, J.H. Leal, T.A. Semelsberger, C.S. McEnally, L.D. Pfefferle, et al., A simple, solvent free method for transforming bioderived aldehydes into cyclic acetals for renewable diesel fuels, Sustainable Energy Fuels 2 (2018) 2742-2746.

[18] M.A. Arellano-Treviño, D. Bartholet, A.T. To, A.W. Bartling, F.G. Baddour, T.L. Alleman, et al, Synthesis of butyl-exchanged polyoxymethylene ethers as renewable diesel blendstocks with improved fuel properties, ACS Sustainable Chem. Eng. 9 (2021) 6266-6273.

[19] R.J. Gill, D.B. Olson, Estimation of soot thresholds for fuel mixtures, Combust. Sci. Technol. 40 (1984) 307-315.

[20] L. Li, P.B. Sunderland, Smoke points of fuelfuel and fuel-inert mixtures, Fire Safety J. 61 (2013) 226-231.

[21] C.S. McEnally, Y. Xuan, P.C. St. John, D.D. Das, A. Jain, S. Kim, et al., Sooting tendencies of cooptima test gasolines and their durrogates, Proc. Combust. Inst. 37 (2019) 961-968.

[22] C.S. McEnally, D.D. Das, L.D. Pfefferle, Yield Sooting Index database volume 2: Sooting tendencies of a wide range of fuel compounds on a unified scale, (2017),

https://doi.org/10.7910/DVN/7HGFT8.

[23] J. Gau, D. Das, C. McEnally, D. Giassi, N. Kempema, M. Long, Yale Coflow Burner Information and CAD Drawings, (2017), https://doi.org/10.6084/m9.figshare.5005007.v1. [24] B. Franzelli, M. Roussillo, P. Scoflaire, J. Bonnety, R. Jalain, T. Dormieux, et al., Multidiagnostic soot measurements in a laminar diffusion flame to assess the ISF database consistency, Proc. Combust. Inst. 37 (2019) 1355-1363.

[25] D. Mathieu, R. Bouteloup, Reliable and versatile model for the density of liquids based on additive volume increments, Industrial \& Engineering Chemistry Research 55 (50) (2016) 12970-12980. 
[26] C.L. Yaws, Yaws' Handbook of Physical

Properties for Hydrocarbons and Chemicals, (2008), https://app.knovel.com/hotlink/toc/id:kpYHPPHC0B /yaw s-handbook-physical/yaws-handbook-physical. [27] J.R. Rumble, CRC Handbook of Chemistry and Physics, 101st Edition, (2019),

http://hbcponline.com/faces/contents/ContentsSearch .xhtml

[28] DIPPR Project 801 - Full Version, Design Institute for Physical Property Research/AIChE, (2019),

https://app.knovel.com/hotlink/toc/id:kpDIPPRPF7/d ippr- project-801-full/dippr-project-801-full.

[29] D.C. Montgomery, Design and Analysis of Experiments, $8^{\text {th }}$ Ed., John Wiley \& Sons, Hoboken, NJ, U.S.A., 2013.

[30] D.D. Das, P.C.S. John, C.S. McEnally, S. Kim, L.D. Pfefferle, Measuring and predicting sooting tendencies of oxygenates, alkanes, alkenes,

cycloalkanes, and aromatics on a unified scale, Combust. Flame 190 (2018) 349-364.

[31] M.S. Graboski, L.M. Robert, Combustion of fat and vegetable oil derived fuels in diesel engines,

Progress in energy and combustion science 24 (2) (1998) 125-164.

[32] O. Herbinet, W.J. Pitz, C.K. Westbrook, Detailed chemical kinetic oxidation mechanism for a biodiesel surrogate, Combustion and Flame 154 (3) (2008) 507-528. 